\title{
Certification et recertification, un nouveau défi pour l'université médicale
}

Jacques ROLAND*

\section{|}

I est curieux de constater qu'en France on s'est posé le problème de la recertification, processus qui permet de constater que les connaissances d'un médecin restent valides et adaptées, avant celui de la certification. Tout est venu en 1996 de l'obligation légale de la formation continue des médecins, donc de la nécessité d'évaluer les résultats de cette formation ; fallait-il reconnaître simplement les efforts de formation, ou fallait-il apprécier une compétence et en fonction de quels critères ? Q uoi qu'il en soit, tout le monde s'en préoccupe, que ce soit les organismes de sécurité sociale, l'O rdre des M édecins, les syndicats ou les associations de formation continue.

\section{Certification}

Si I'on parle assez facilement de recertification, terme commode, le concept de certification n'est pas luimême établi à la fin des études de médecine. La référence ne peut être que professionnelle, il s'agit d'abord de posséder des connaissances pertinentes et applicables dans la profession choisie. II s'agit ensuite d'être capable de mettre en œuvre ces connaissances sous forme d'aptitudes et de décisions, il s'agit enfin de le faire dans le cadre d'un comportement adapté.

Certaines disciplines, en particulier chirurgicales, sont entrées dans ce processus, en organisant des boards dont le modèle est généralement européen. $M$ ais on devine la complexité d'une telle évaluation, et I'on craint les dérives dans lesquel les nous risquons de nous enfoncer.

- La première difficulté vient de la mise au point d'un système de référence. $0 \mathrm{n}$ a cru trouver la solution par les conférences de consensus. En France, I'A.N .D.E.M ., puis I'A.N .A.E.S. (notre agence d'évaluation des pratiques) ont produit des textes de qualité, mais naturellement sans pouvoir prétendre à l'exhaustivité, ni être en mesure de réviser périodiquement ces mêmes textes. Le concept d'Evidence Based M edecine a permis, lui aussi, la publication d'ouvrages utiles, mais dont on perçoit, là encore, les limites. Le système de référence demeure donc encore essentiellement dans le bagage intellectuel des enseignants...

- Une ambition souvent exprimée est d'établir un contrôle en fin de formation. Ce contrôle serait assuré par un jury composé essentiellement d'universitaires et de professionnels concernés, extérieurs à l'établissement. U ne telle procédure devrait permettre de juger le candidat dans des conditions qui sont proches de son exercice. Les E.C.O.S. en sont une approche, dont on connaît la pertinence, mais également le coût, l'investissement en temps et l'impossible exhaustivité.

\footnotetext{
*Président de la Conférence des Doyens des Facultés de Médecine Françaises

Correspondance : Pr Jacques Roland - Faculté de Médecine de Nancy - BP 184 - 54505 Vandoaivre les Nancy cedex Tél. + 33383592501 - Télécopie : + 33383592503 - Adresse électronique : roland@facmed.u-nancy.fr
} 
Ils commencent à s'implanter dans les Facultés françaises dans le troisième cycle de médecine générale. $M$ ais le système des malades simulés, dont on comprend l'utilité pour une standardisation des épreuves, est critiquable pour plusieurs raisons. La première est qu'ils sont plus nécessaires pour classer des étudiants que pour juger des aptitudes de chacun ; la deuxième est que ce système favorise la tendance perverse à pré parer des épreuves centrées sur des fictions totales, plus que de mettre toute notre attention sur l'approche des malades réels et la résolution de leurs vrais problèmes. Certains pédagogues affirment même sans se troubler qu'ils sont plus intéressants dans le processus éducationnel que des malades réels! N e nions pas les qualités de cette approche, mais gardons-nous d'en faireune religion.

- Les stages, qu'ils soient hospitaliers ou dans le cabinet d'un médecin sont certainement les terrains les plus adaptés à l'observation des aptitudes d'un jeune médecin. II s'agit d'un contrôle continu, en situation de pratique réelle, avec des vrais malades. Seule la formulation les éloigne actuellement de la certification. N ous devons cependant garder en mémoire les imperfections actuelles, et leur difficile objectivité.

\section{Recertification}

La société ne peut que souhaiter le maintien de l'aptitude de ses médecins, mais pas à n'importe quel prix. Le système devrait être à la fois supportable économiquement, adapté à l'activité professionnelle, respectueux des personnes et pertinent dans ses résultats. $C^{\prime}$ 'est dire qu'il faut des adaptations individuelles, et que plusieurs formules doivent être proposées. 0 n peut également souhaiter que l'ensemble des partenaires de l'éducation médicale participe à l'organisation et à la mise en pratique de la recertification. Syndicats, associations de formation continue, 0 rdre des médecins, sociétés savantes, et naturellement Université, doivent travailler ensemble sur ce sujet.
C'est ainsi que le processus sera accepté et supporté par tous les médecins, et qu'il sera viable.

O n ne peut qu'évoquer aussi, à la faveur de cette réflexion sur la recertification, la nécessaire mise en place de « passerelles » entre les métiers médicaux. Si I'on désire favoriser la possibilité de changements de carrière de certains d'en tre nous, cela passera obligatoirement par un processus analogue à celui de la recertification.

\section{Conclusion}

Certification et recertification ne peuvent échapper à l'attention des facultés de médecine. Ce thème a été évoqué à une réunion plénière de la $C$ onférence Internationale des $D$ oyens des Facultés de $M$ édecine d'Expression Française. M ais le débat ne peut s'y limiter, il nécessite un esprit d'ouverture très large vers les organisations professionnelles, c'est dire l'importance primordiale du défi que nous devons relever. 